\title{
Téoros
}

Revue de recherche en tourisme

\section{La gestion du marketing touristique : une perspective empirique}

\section{Pierre Filiatrault, Jean Charles Chebat et Carole Marcoux}

Volume 5, numéro 3, novembre 1986

La gestion touristique : les nouvelles technologies

URI : https://id.erudit.org/iderudit/1080539ar

DOI : https://doi.org/10.7202/1080539ar

Aller au sommaire du numéro

Éditeur(s)

Université du Québec à Montréal

ISSN

0712-8657 (imprimé)

1923-2705 (numérique)

Découvrir la revue

Citer cet article

Filiatrault, P., Chebat, J. C. \& Marcoux, C. (1986). La gestion du marketing touristique : une perspective empirique. Téoros, 5(3), 30-33.

https://doi.org/10.7202/1080539ar d'utilisation que vous pouvez consulter en ligne. 


\title{
La gestion du marketing touristique: une perspective empirique
}

\author{
par Pierre Filiatrault, Jean Charles Chebat et Carole Marcoux*
}

\section{Introduction}

Peu de recherches empiriques ont traité de la planification du marketing et des pratiques de gestion des entreprises de services, et plus particulièrement de celles de l'industrie touristique. La documentation sur le marketing comprend des guides d'instruction expliquant comment préparer un plan de marketing (Bradway et al. 1982; Makens, 1986; Rice, 1984; Stevens, 1982), des rapports traitant ou bien de la pratique de la planification d'entreprise par les compagnies et dirigeants en général (Boulton et al., 1982; Snyder et Glueck, 1980), ou bien de descriptions de l'organisation et des procédures impliquées dans la planification du marketing (John et Martin, 1984; Paul, 1981; Stasch et Lanktree, 1980). Filiatrault et Chebat (1986) ainsi que Gronroos (1983b) figurent parmi les rares chercheurs à s'être intéressés aux pratiques de gestion du marketing de services.

Lorsqu'il s'agit du tourisme, les écrits sont encore plus limités, consistent principalement en des descriptions normatives et se concentrent sur l'industrie du voyage touristique (Rovelstad et Roff Blazer, 1983) ou sur le marketing d'une région ou d'un pays particulier. La planification du marketing et les pratiques de gestion des entreprises touristiques ont reçu peu d'attention; seules quelques exceptions ont pris une approche de stratégie de marketing (Zaccolo, Scotton et Jeresko, 1983). Le but de cet article est de présenter les résultats d'une recherche exploratoire sur la planification du marketing et sur les pratiques de gestion dans l'industrie touristique.

\section{Méthodologie}

Le cadre échantillonal de l'étude est constitué d'entreprises situées au Québec et listées chez Dunn \& Bradstreet. Une procédure d'échantillonnage aléatoire nous a servi à selectionner 2500 entreprises de

\footnotetext{
- Pierre Filiatrault est directeur du Centre de recherche en gestion de I'UOAM, Jean-Charles Chebat est titu laire de la chaire de marketing John-Labatt de I'UOAM: tous deux sont profesgeurs titulaires au département des sciences administratives. Carols Marcoux termine son bacc-alaurétat en administration à PUQAM. Cet article est une adaptation de la communication presentee au corigres annuel de la "Travel and Tourism Fiesearch Association'", Memphis, Tenn., juin 1996.
}

services. Un questionnaire, accompagné d'une lettre couverture, fut posté au responsable du marketing de chacune de ces entreprises. Au total, 320 questionnaires furent retournés; 293 furent retenus pour analyse: 46 entreprises de services touristiques et 247 entreprises d'autres services (vente au détail, finance, etc.). La plupart des questionnaires rejetés concernaient des répondants qui oeuvraient à la fois dans les biens et les services.

Le questionnaire se divisait en quatre blocs. Dans le premier bloc, les répondants décrivaient leur entreprise en termes de type d'industrie, de chiffres d'affaires, du nombre d'employés, etc. Dans le deuxième bloc, les caractéristiques organisationnelles du service du marketing étaient mises en évidence. Les dirigeants faisaient part de la planification du marketing ainsi que des activités et pratiques de gestion de leur entreprise dans le troisième bloc. Le quatrième et dernier bloc sondait quelques caractéristiques socio-démographiques et psychologiques des répondants.

\section{Résultats}

La présente section comprend trois volets: a) une analyse comparative des caractéristiques opérationnelles des entreprises touristiques et de d'autres entreprises de services; b) une description des pratiques de planification du marketing liées aux caractéristiques opérationnelles des entreprises ainsi qu'à l'influence que le marketing y exerce; c) et une analyse de certaines activités de gestion du marketing.

\section{Analyse comparative des caractéristiques opérationnelles}

Les firmes classées dans l'industrie touristique incluent les hôtels, restaurants et organisations de loisir. Les autres entreprises de services oeuvraient dans les transports, le commerce au détail, la finance, les assurances, l'immobilier, les services professionnels, les soins personnels, etc. $L e$ revenu annuel des entreprises touristiques est, de façon significative, plus bas $(p=$ .001) que les autres entreprises de services (tableau 1). Au total, 12 firmes touristiques $(27,3 \%)$ avaient un chiffre d'affaires

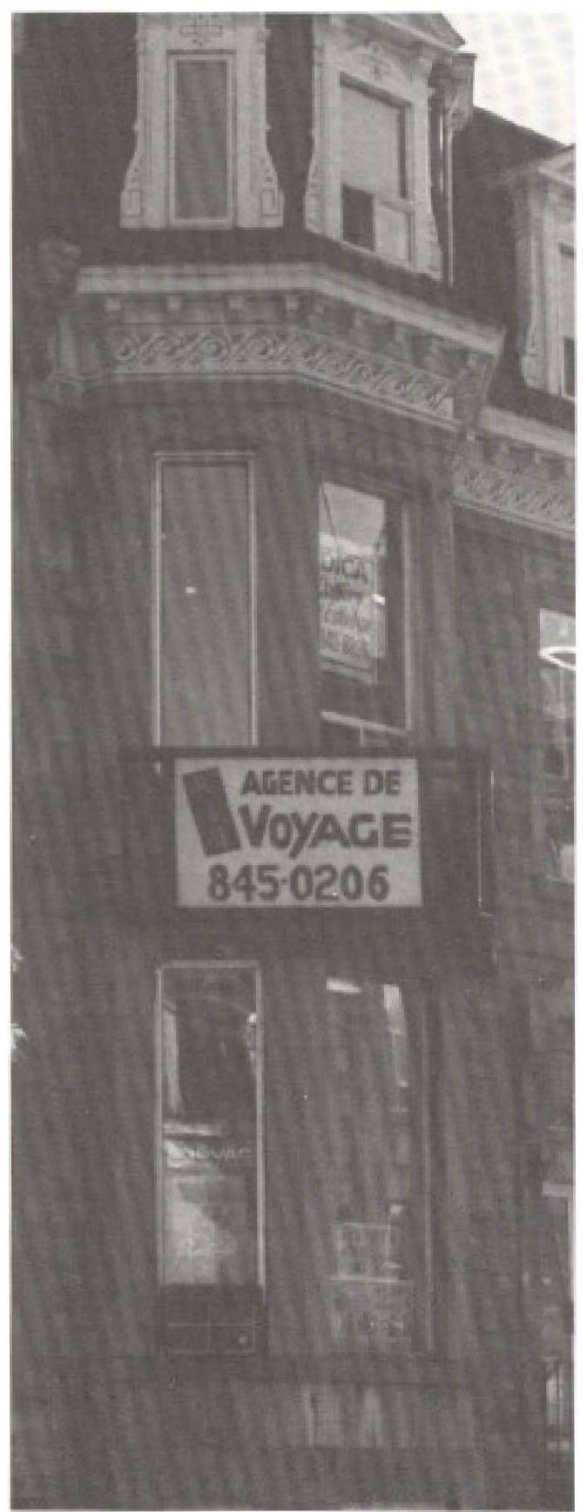

Des entreprises de services

de moins d'un million de dollars alors que $40,2 \%$ des autres entreprises de services avaient des revenus de 10 millions ou plus. Près de la moitié $(44,5 \%)$ de toutes les entreprises touristiques employaient moins de cinquante personnes, alors que le reste des compagnies de services avaient tendance à embaucher 250 employés ou plus. Les entreprises de services du milieu tou- 
ristique possédaient un conseil d'adminis= tration moins fréquemment $(63,0 \%)$ que celles des autres secteurs $(74,5 \%)$. Les résultats financiers étaient jugés moins satisfaisants dans les organisations touristiques, cependant cette différence n'était pas statistiquement significative. Finalement, il n'y avait aucune difference significative entre les deux types d'entreprises lorsqu'il s'agissait du pourcentage du chiffre d'affaires accorde aux dépenses de marketing: $38,1 \%$ des entreprises de services en général allouaient $2 \%$ ou moins à leur budget de marketing contre $33,1 \%$ pour les entreprises touristiques; plus d'un tier $(35,6 \%)$ de celles-ci accordaient $6 \%$ ou plus à ces mêmes dépenses.

\section{La planification du marketing}

Les entreprises touristiques s'engageaient dans la planification du marketing aussi souvent que les autres types d'entreprises de services: $65,0 \%$ des entreprises touristiques préparaient un plan de marketing annuel. Parmi les répondants de l'industrie touristique, les firmes qui étaient plus petites (par rapport au chiffre d'affaire $(\mathrm{p}=, 05)$ et au nombre d'employés $(\mathrm{p}=, 05))$ avaient tendance à préparer un plan annuel de marketing moins souvent que les grandes entreprises. Les organisations qui préparaient un plan de marketing annuel étaient celles qui avaient un conseil d'administration $(\mathrm{p}=, 05)$ et celles dont le pourcentage du chiffre d'affaires des dépenses de marketing se trouvait plus élevé, quoique ces résultats n'étaient pas statistiquement significatifs.

Les dirigeants des entreprises touristiques percevaient, dans $67,5 \%$ des cas, que I'influence de la fonction marketing demeurait stable ou avait légèrement augmenté au cours des cinq derniêres années; $87,5 \%$ de l'ensemble des dirigeants croyaient que I'influence dans l'entreprise de cette même fonction augmenterait au cours des cing prochaines années. Les firmes qui prévoyaient une hausse de cette influence avaient plus tendance, et ce de façon significative, à préparer un plan annuel de marketing $(\mathrm{p}=, 001)$.

\section{Activités de gestion du marketing}

La planification du marketing représente une tâche stratégique importante normalement effectuée en coopération avec les autres services de l'entreprise. Par contre, la gestion du marketing prend la forme de nombreuses actions tangibles. Pour le dirigeant de marketing, ces activités définissent la nature de la fonction marketing. Afin de découvrir le niveau d'activité de marketing des entreprises de notre bchantillon, nous avons sélectionné et analysé un certain nombre de pratiques de marketing lices au personnel de ce service ainsi qu'aux diverses activités traditionnelles de communication.
TABLEAU 1

Analyse des caracteristiques operationnelles des entreprises touristiques et de services

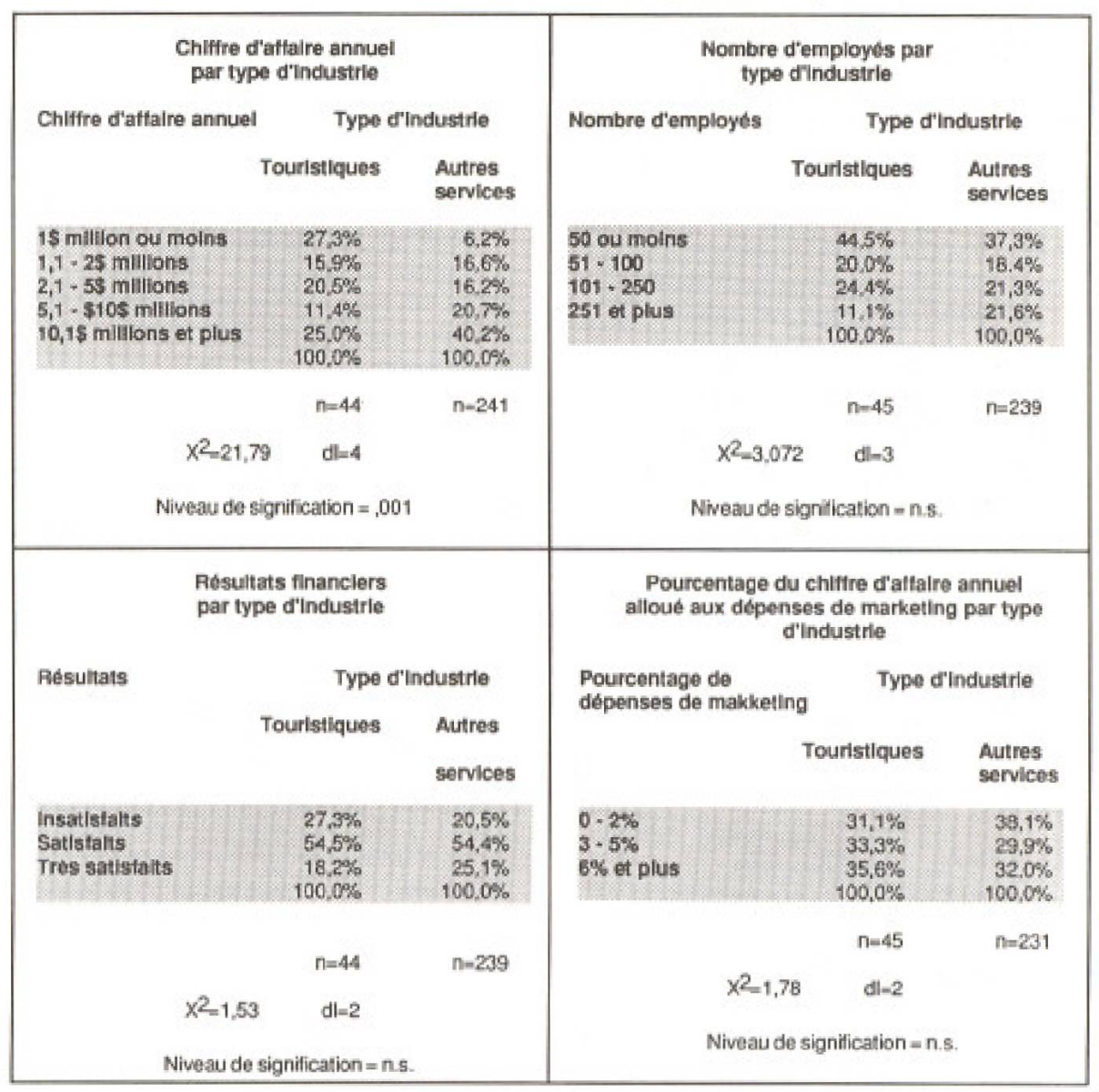

TABLEAU 2

Actlvite de gestion du marketing a I'intrant par pratlquo de planificatlon du marketing

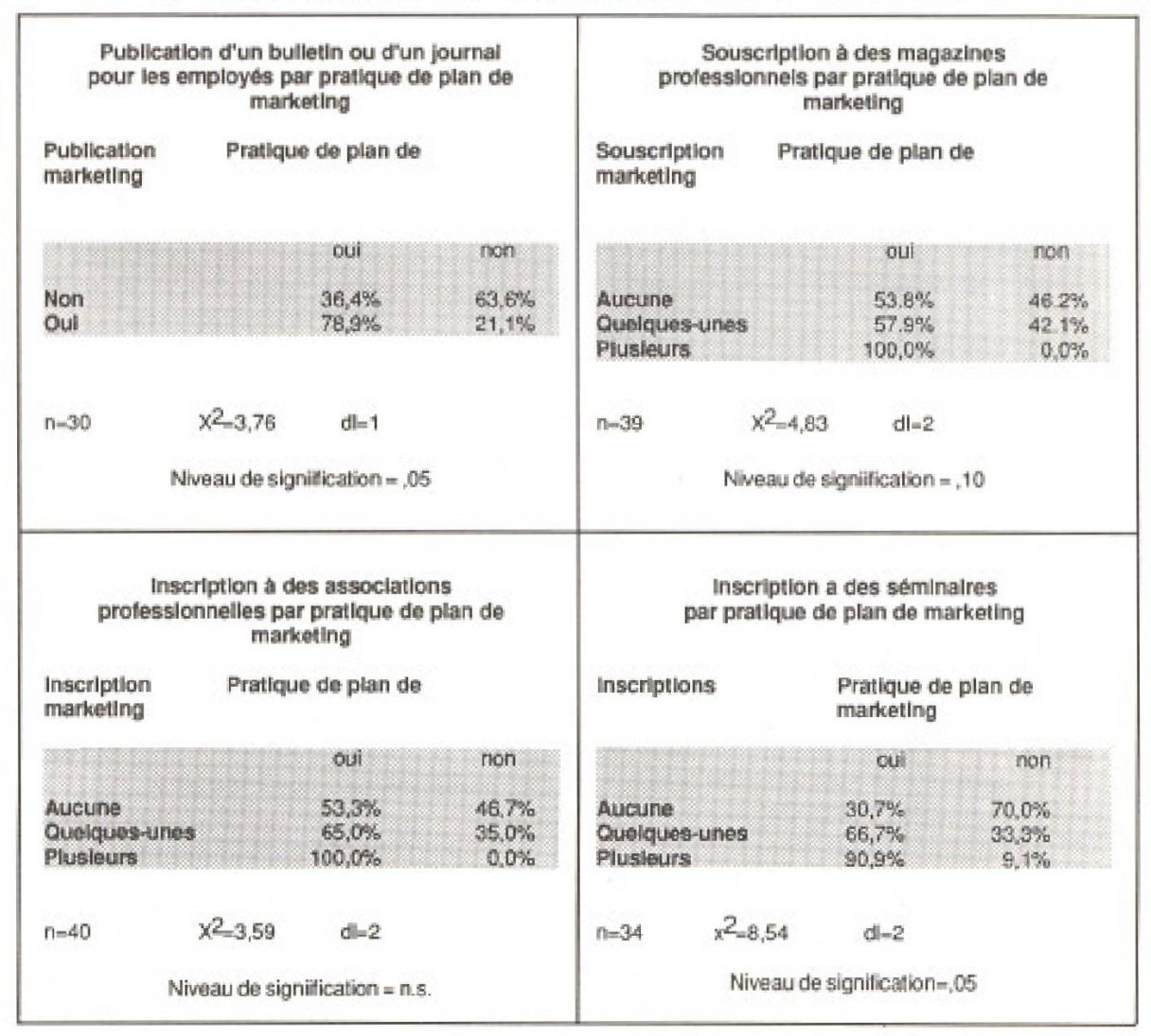




\section{Activités de gestion du marketing à l'intrant}

La gestion des ressouroes humaines al l'intérieur d'un service de marketing peut prendre plusieurs formes. Ceci peut comprendre des activités internes de marketing telles la publication d'un bulletin ou d'un journal pour les employés. Cela peut aussi inclure le développement personnel par l'entreprise d'abonnements à des magazines professionnels, d'inseriptions à des associations professionnelles, à des séminaires ou programmes de formation (tableau 2).

Les entreprises qui effectuaient un plan annuel de marketing étaient aussi celles qui, plus probablement $(\mathrm{p}=, 05)$, publiaient un bulletin ou un journal pour les employes. Ces mêmes entreprises paraissaient plus conscientes du développement de leurs employés de marketing. Elles souscrivaient plus souvent à des revues professionnelles $(\mathrm{p}=, 10)$, inscrivaient plus fréquemment le personnel à des associations professionnelles, bien que cette difference ne soit pas statistiquement significative, et enregistraient leurs employés à des séminaires ou programmes de formation plus souvent $(\mathrm{p}=, 05)$.

\section{Activités de gestion du marketing à l'extrant}

Les activités de communication plus traditionnelle fournissent également un indice du niveau d'activité de marketing. Elles sont les extrants d'un processus qui inclut le développement organisationnel, les êtudes de marche, la détermination du budget ainsi que la planification du marketing qui définit les stratégies et leurs moyens d'implantation.

Les tableaux 3 et 4 présentent les activités de gestion du marketing les plus courantes à l'extrant. Les activités de promotion les plus fréquentes sont les publications de catalogues ou brochures et de listes de prix. Mais seulement $43,3 \%$ des répondants ont participé à des expositions en tant qu'exposants. Le niveau d'activités promotionnelles des entreprises touristiques était similaire à celui des autres entreprises de services; cependant la participation à des expositions était quelque peu faible $(\mathrm{p}=, 10)$. La majorité des entreprises $(92,1$ \%) avaient recours a la publicité dans les journaux et $82,9 \%$ des entreprises annoncaient aussi à la radio et à la télévision. La publicité dans les revues était moins fréquente, mais $91,7 \%$ des répondants réalisaient des activités de relations publiques. Le niveau d'activités de publicité et de relations publiques était un peu plus élevé dans les entreprises touristiques que dans les autres entreprises de services. Il était significativement plus élevé dans les entreprises touristiques dans le cas de la radio et télévision $(\mathrm{p}=, 01)$, des journaux $(\mathrm{p}=, 10)$ et des revues populaires destinées aux consommateurs $(\mathrm{p}=, 05)$; le niveau

TABLEAU 3

Actlvite de gestlon du marketing at Fextrant

(actlvite de promotion) par pratique de planification du marketing

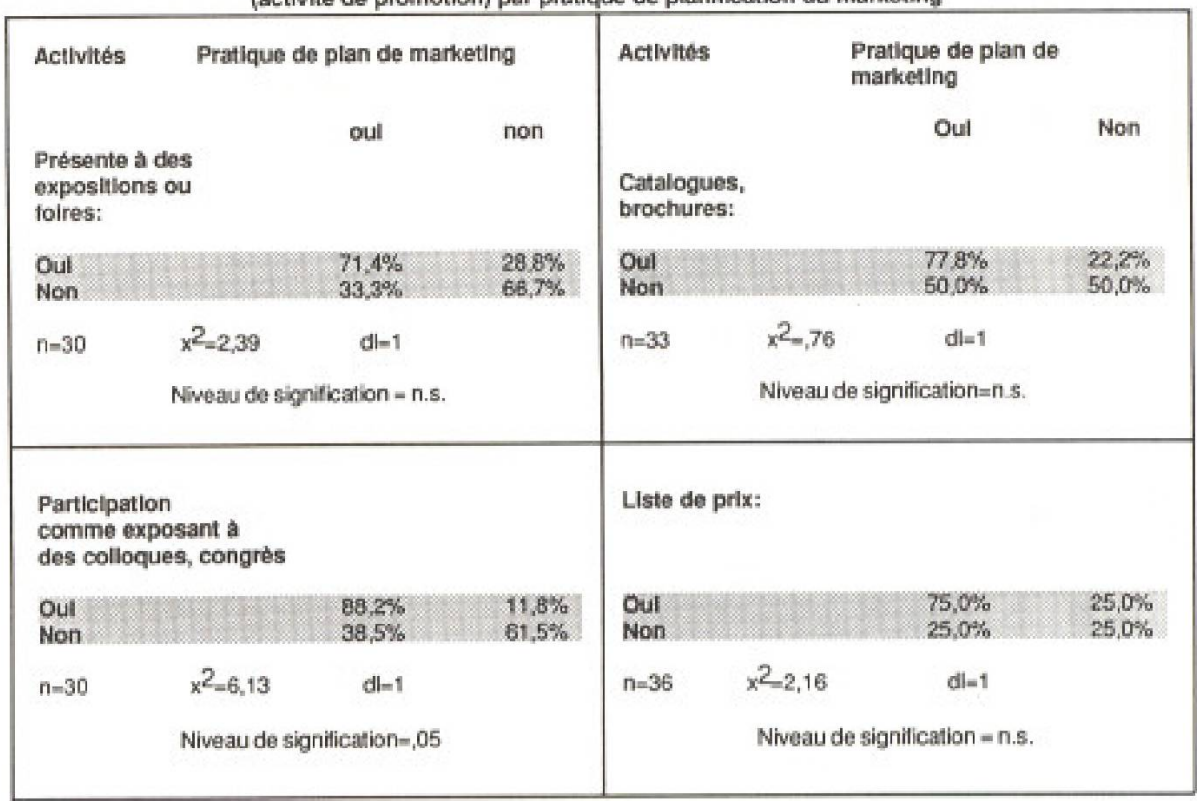

TABLEAU 4

Actlitte de gestlon du marketing a lextrant (publiche et relatlons publiques) par pratlque de planiflcation du marketing

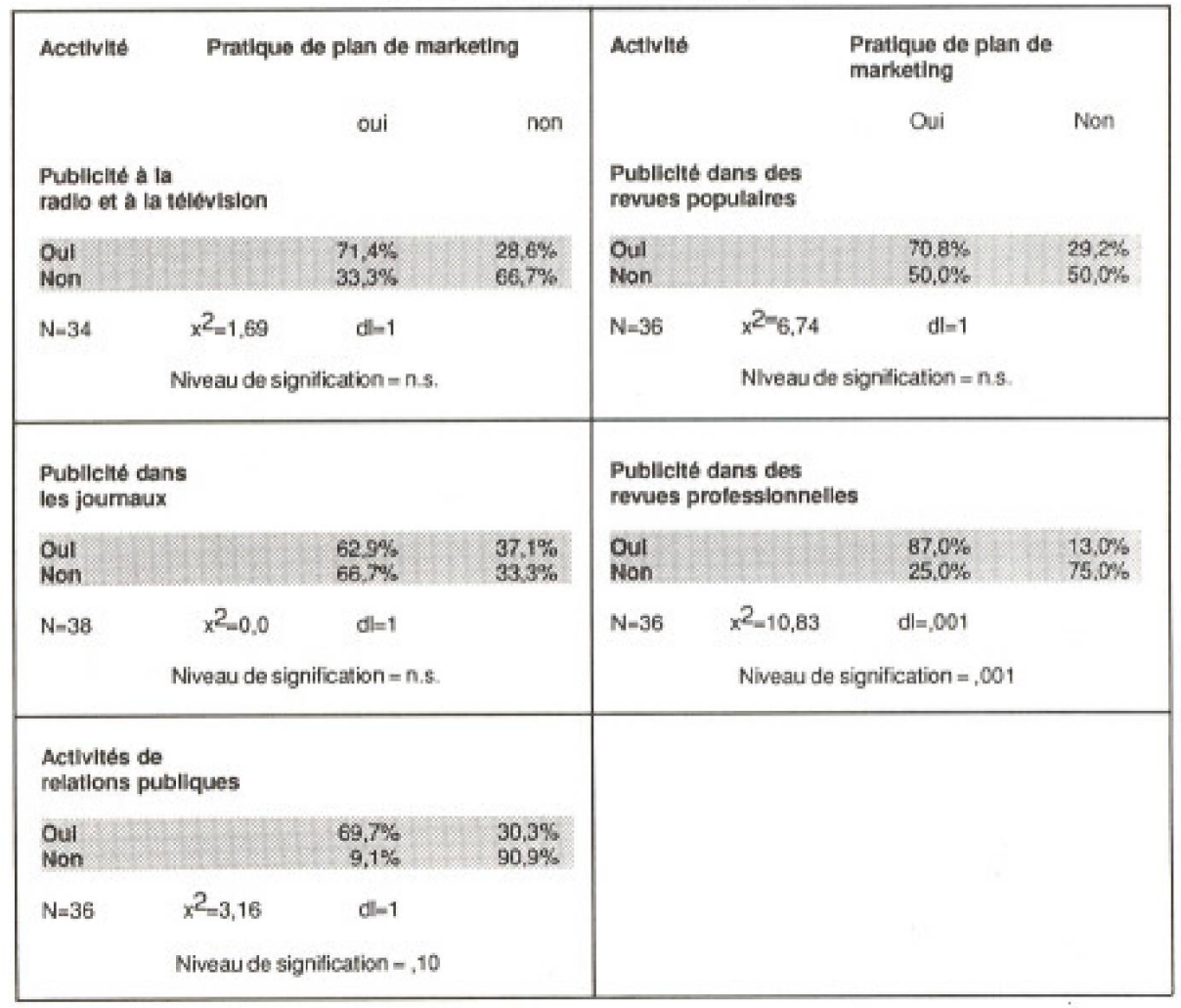

d'activités était toutefois similaire dans les revues professionnelles, de mêtne que pour les relations publiques.

Généralement, les entreprises touristiques qui préparent un plan annuel de marketing tendent à s'engager plus intensếment que les autres firmes touristiques dans les activites de marketing; cependant, la différence n'est pas toujours statistiquement significative. Approximativement les trois quarts des entreprises qui préparaient un plan de marketing, s'engageaient dans des activités promotionnelles. La participation comme exposant à des congrès ou colloques offre néanmoins la seule différence significative $(\mathrm{p}=, 05)$. Comparativement, les firmes qui ne préparaient pas de plan 
de marketing s'avèraient plus dynamiques dans les activités de publicité que dans les autres activités, mais encore là, n'égalaient pas celles qui planifiaient. La publicité dans les journaux se retrouvait fréquemment dans les deux types d'entreprises. Toutefois, les firmes préparatrices d'un plan publiaient plus souvent leur publicité dans les revues professionnelles $(p=, 001)$ et $s$ 'aventuraient plus fréquemment dans des activités de relations publiques $(\mathrm{p}=, 10)$.

\section{Conclusion}

L'intégration du marketing dans les entreprises de services doit avoir comme principe fondamental le concept de marketing. Mais cette philosophie de gestion basée sur les relations d'échanges est appliquée par des praticiens dans des activités et pratiques concrètes. Il y a eu peu de recherches empiriques de cette application dans les entreprises de services en général, et plus particulierement dans les entreprises de services touristiques. La présente recherche exploratoire avait comme objectif d'êtudier cette problématique.

Une analyse comparative des caractéristiques des entreprises de services touristiques et des autres entreprises de services fut conduite. Les firmes touristiques s'avèrent plus petites en termes de chiffres d'affaires et de nombre d'employés. Les résultats financiers et le pourcentage du chiffre d'affaires alloué aux dépenses de marketing se rejoignaient dans les deux groupes.

Les deux catégories d'entreprises de service préparaient des plans annuels de marketing, mais les petites firmes touristiques le faisaient moins souvent. Les organisations dont le pourcentage des revenus accordé aux dépenses de marketing était plus élevé se révélaient plus enclines à préparer un plan annuel de marketing, quoique ceci ne soit pas statistiquement significatif. Quoiqu'il en soit, les firmes prévoyant une plus grande influence de la fonction marketing sur leur gestion au cours des cinq prochaines années s'engageait de façon significative plus souvent dans la préparation de plans de marketing. Des activités de gestion du marketing à l'intrant et à l'extrant furent aussi étudiées. Les entreprises préparatrices d'un plan avaient plus tendance à pratiquer une activité interne traditionnelle de marketing: la publication d'un bulletin. Elles souscrivaient aussi plus facilement à des revues professionnelles et inscrivaient plus aisément leurs employés à des sếminaires, programmes de formation et associations professionnelles. Généralement, les organisations préparant un plan s'aventuraient plus aisément dans des activités de promotion et de relations publiques. Ces firmes se montraient plus actives en publicité, quoique la plupart des entreprises utilisaient intensément la publicité dans les journaux, qu'elles aient ou non un plan de marketing.
Une revue de littérature du marketing a révélé peu de recherches empiriques sur les pratiques administratives de marketing, en particulier en ce qui a trait à l'industrie des services. En conséquence, nous avons entreprit une recherche exploratoire sur la planification du marketing et les activités de gestion. Même si le nombre de répondants appartenant à l'industrie touristique de services était peu élevé, nous avons obtenu des résultats empiriques qui permettent de discerner quelques aspects de ce sujet négligé. Nous espérons que ces résultats fourniront aux praticiens de marketing lies aux entreprises de services touristiques, une meilleure compréhension de leur tâche, et qu'elles encourageront les chercheurs a explorer plus largement la planification du marketing touristique et les activités de gestion. $f$

\section{Bibliographie}

BOULTON, William, R., Stephen G. Franklin, William M. Lindsay and Leslie W. Rue, (1982) "How are Companies Planning Now? - A Survey', Long Randge Planning, vol. 15 , no. 1 , pp. 82-6.

BRADWAY, Bruce M., Robert E. Pritchard and Mary Anne Frenzel (1982), Strategic Marketing, A Handbook for Managers, Business Owners and Entrepreneurs, Reading Mass: Addison Wesley Pub. Co.

BUTTLE, Francis, (1985), "'The Marketing Strategy Worksheet. A Practical Planning Tool", Long Range Planning, vol. 18, no. 4, pp. $80-8$.

FILIATRAULT, Pierre et Jean-Charles Chebat (1986), "Services Marketing Management Practices and Activities: A Comparative Analysis". Document de trvail no 24-86, Centre de recherche en gestion, Université du Québec à Montréal.

GOETSCH, H.W. (1979), How to Prepare and Use Marketing Plans for Profit, Chicago: American Marketing Association.

GRONROOS, Christian (1983a), "Innovative Marketing Strategies and Organization Structures for Service Firms", in Emerging Perspectives on Services Marketing, eds. Leonard L. Berry, G., Lynn Shostack and Gregory D. Upah, Chicago, American Marketing Association, 9-21.

GROONROS, Christian (1983b), Strategic Management and Marketing in the Service Sector, Cambridge Mass.: Marketing Science Institute.

HOPKINS, David S. (1981), The Marketing Plan, Report no 801, New York: The Conference Board.
JOHN, George and John Martin (1981), "Effects of Organizational Siructure of Marketing Planning on Credibility and Utilization of Plan Outpout', Journal of Marketing Research, vol. 21, no, 2, (May), 170-83.

LUTHER, W.M. (1982), The Marketing Plan: How to Prepare and Implement It, New York, American Management Association.

MAKENS, James C. (1985), The Marketing Plan Workbook, New York, Prentice-Hall, Inc.

PAUL, Ron (1981), "Organizing for Mar. keting Planning ", Sales and Marketing Management, vol. 122 , no. 8 (December), pp. 37-9.

RICE, Craig S. (1984), Marketing Planning Strategies, A Guide to Small or Medium-Sized Companies, Chicago, The Dartnell Corporation.

ROVELSTAD, James M. and Suzanne Roff Blazer (1983), "Research and Strategic Marketing in Tourism: A Status Report", Journal of Travel Research, vol. 18, (Fall), pp. 2-7.

SNYDER, Neil and William F. Glueck (1980), "How Managers Plan - The Analysis of Managers 'Activities", Long Range Planning, vol. 13 (February), pp. 70-6.

STASCH, Stanley F. and Patricia Lanktree (1980), "Can your Marketing Planning Procedures Be Improved?" Journal of Marketing, vol. 44 (Summer), pp. 79-90.

STEVENS, Robert E. (1982), Strategic Marketing Plan Guide, Englewood Cliffs N.J., Prentice-Hall, Inc.

ZACCOLO, Ronald L., Donald WA. Scotton and David A. Jeresko (1983), "Strategic Market Planning in the Commercial Airline Industry", Journal of the Academy of Marketing Science, vol. 11, no. 4 (Fall), pp. 404-16. 